\title{
Neuroinflammatory gene expression in chronic prostatitis/ chronic pelvic pain syndrome patients: insights into etiology and phenotype biology
}

\author{
Daniel A. Shoskes, Karen S. Keslar, Paige Gotwald, Ryan Berglund, Sarah Vij \\ Glickman Urological and Kidney Institute, The Cleveland Clinic, Cleveland, OH, USA \\ Contributions: (I) Conception and design: DA Shoskes, KS Keslar; (II) Administrative support: P Gotwald; (III) Provision of study materials \\ or patients: DA Shoskes, R Berglund, S Vij; (IV) Collection and assembly of data: DA Shoskes, P Gotwald, KS Keslar; (V) Data analysis and \\ interpretation: DA Shoskes, KS Keslar; (VI) Manuscript writing: All authors; (VII) Final approval of manuscript: All authors. \\ Correspondence to: Daniel A. Shoskes. Cleveland Clinic, 9500 Euclid Ave, Desk Q10-1, Cleveland OH, 44195, USA. Email: dshoskes@gmail.com.
}

Background: Chronic prostatitis/chronic pelvic pain syndrome (CP/CPPS) has diverse clinical phenotypes and its etiology is multifactorial. Studies to date of gene expression in humans have been limited to small numbers of target genes. NanoString can simultaneously measure hundreds of genes. We wished to study gene expression in blood and urine of CP/CPPS patients compared to controls for neuroinflammatory genes and characterize the results by patient phenotype.

Methods: Blood and urine were collected from 10 men with CP/CPPS and 7 asymptomatic controls. RNA was isolated from urine pellets using Qiagen RNeasy kits. Whole blood was collected and RNA isolated. $100 \mathrm{ng}$ of RNA was used for gene expression analysis with the 770-gene NanoString Human Neuroinflammation gene panel. Data was imported into Rosalind (OnRamp Bioinformatics) for normalization, calculation of fold-changes and $\mathrm{P}$ values, and identification of enriched pathways. Gene expression was considered significantly different if there was a greater than $1.5 \times$ change compared to controls and corrected $\mathrm{P}$ was $<0.05$.

Results: Mean patient age was 42.2 years, median symptom duration was 15.5 months, median UPOINT domains was 3 and mean total National Institute of Health-Chronic Prostatitis Symptom Index Score was 28.8. In blood, there were 5 genes with significantly different expression to controls, the largest differences found in FOS1 (neuropathic pain control), PROS1 (blood clotting) and DDX58 (antiviral innate immunity). Gene set analysis showed differences in inflammation, angiogenesis and cytokine signaling. In urine there were 48 genes with significantly different expression including SLAMF8 (lymphocyte activation) and LAIR1 (inhibits B and T cell function). Gene set analysis showed differences in carbohydrate metabolism, neurons and neurotransmission, adaptive immunity and inflammatory signaling. Subgroup analysis by UPOINT domain showed unique gene expression in the Organ Specific and Neurologic/Systemic domains in both blood and urine for neurogenic pain and cytokine signaling associated genes

Conclusions: Men with CP/CPPS have a diverse set of neuroinflammatory genes with differential expression compared to controls. Clinical phenotypes have distinct patterns of gene expression. These findings could lead to novel biomarker development, emphasize the importance of multimodal therapy targeting diverse pathways and further validate the biologic basic of clinical phenotyping.

Keywords: Prostatitis; chronic pelvic pain; inflammation

Submitted Apr 30, 2021. Accepted for publication Jul 15, 2021.

doi: $10.21037 /$ tau-21-387

View this article at: https://dx.doi.org/10.21037/tau-21-387 


\section{Introduction}

Chronic prostatitis/chronic pelvic pain syndrome (CP/ CPPS) is a common condition with diverse clinical phenotypes (1). A specific etiology for all patients is elusive, with prior evidence for infective, inflammatory, and neuromuscular pathways (2). Prior attempts to find biomarkers for these etiologic pathways have focused on expression of several inflammatory genes in urine and blood (3), genetic polymorphisms (4) and the mediators of neuropathic pain such as nerve growth factor (NGF) (5). Such attempts have been limited by the techniques employed, which can only look at several genes at a time in one sample.

The NanoString nCounter is a novel technology that allows direct measurement of mRNA expression with a small sample for a large number of genes without the need for conversion to cDNA or polymerase chain reaction (6). A premade cassette has almost 800 genes related to inflammation and neuropathic pain. We therefore wished to study the gene expression in blood and urine for this large number of neuroinflammatory genes in men with CP/CPPS compared to asymptomatic controls. This approach allows the simultaneous measurement of related genes whose key role in pathophysiology may lie more in their orchestrated expression rather than the levels of any one gene at a time. We hypothesize that men with CP/CPPS will have unique RNA expression signatures and that men with different clinical phenotypes may have different pathways active.

\section{Methods}

The study was approved by the Cleveland Clinic IRB (protocol 19-1515) and appropriate written consent was obtained on all subjects. The study conformed to the provisions of the Declaration of Helsinki (as revised in 2013). Blood and midstream urine were collected from 10 men presenting to a specialty prostatitis clinic with CP/CPPS using the National Institute of Health (NIH) definition of category III (7) as well as from 7 asymptomatic controls presenting for a vasectomy between January and December 2020. Symptom severity was assessed with the NIH-Chronic Prostatitis Symptoms Index (8) and clinical phenotype documented with UPOINT (9).

RNA was isolated from urine pellets using Qiagen RNeasy kits. Whole blood was collected and RNA isolated using the Tempus Spin RNA isolation kit. $100 \mathrm{ng}$ of RNA was used for gene expression analysis with the 770-gene NanoString Human Neuroinflammation gene panel.

\section{Statistical analysis}

Data was imported into Rosalind (OnRamp Bioinformatics) for normalization, calculation of fold-changes and $\mathrm{p}$ values, and identification of enriched pathways. MultiDimensional Scaling (MDS) graphs were generated for individual samples. Gene expression was considered significantly different if there was a greater than $1.5 \mathrm{x}$ change compared to controls and $\mathrm{P}$ was $<0.05$ corrected for multiple comparisons.

\section{Results}

Of the 10 men with CPPS, the mean age was 42.2 years (range 22-59 years) and median symptom duration 15.5 months (range 3 months to 35 years). Using clinical phenotyping there were a median of 3 UPOINT domains positive (range 2-4) however no patients had evidence for infection ("I" domain) and only 1 patient didn't have bothersome urinary symptoms ("U" domain). The mean total NIH-CPSI score was 28.8 (range, 14-39). These values are all typical for CPPS patients we have studied and reported on in the past (10). The control asymptomatic men had a mean age of 42.6 years (range, $30-55$ years).

Comparing gene expression between CP/CPPS and control in blood showed moderate differences. The multidimensional scaling (MDS) plot showed broad overlap in all samples (data not shown). There were only 4 genes with expression $>1.5 \times$ different in CP/CPPS $v s$. controls and a $\mathrm{P}$ value $<0.05$ (Figure 1), with 1 being lower in CP/ CPPS and 3 being higher (see Table 1 for gene names and function). The only marginally significant gene set analysis difference between groups was for inflammatory signaling and NF-kB [significance score (SS) 1.11]. By contrast there were more differences seen for the UPOINT phenotypes that had sufficient heterogeneity in the sample. In our patient cohort nobody had the I domain (infection), only 1 didn't have the U domain (urinary) and only 1 didn't have the $\mathrm{T}$ domain (tenderness). As seen in Figure 2, the MDS plots for yes or no for Organ Specific, Psychosocial, and Neurogenic/Systemic all showed evidence for grouping. For Organ Specific (yes vs. no) there were 16 genes with significantly different expression led by BCL2A1 ( $-2.1 \times$ change, $\mathrm{P}=0.03)$, CST7 $(-1.9 \times$ change, $\mathrm{P}=0.03)$ and IL1B $(-1.9 \times, P=0.04)$. By gene set analysis grouping 


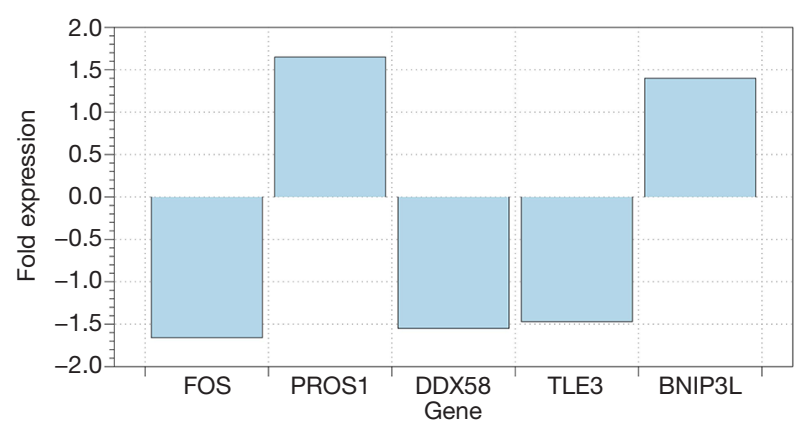

Figure 1 Mean differential gene expression between chronic prostatitis/chronic pelvic pain syndrome patients and controls in blood. Genes listed had at least $1.5 \times$ differential expression with $\mathrm{P}<0.05$. For full gene function please see Table 1. FOS FBJ murine osteosarcoma viral oncogene homolog, PROS1 Protein S (alpha), DDX58 Dead Box polypeptide 58 (also called RIG-1), TLE3 Transducer like enhancer of split 3, BNIP3L BCL2/adenovirus E1B interacting protein.

there were differences in carbohydrate metabolism (SS 1.4), lipid metabolism (SS 1.3) and NF-kB expression (SS 1.3). Neurologic/Systemic had no genes with differential expression and Psychogenic had only 1 and gene set analysis showed no clear pathway clusters.

By contrast, gene expression between CP/CPPS and control urine samples showed more differences in extent and degree. MDS plots showed clear segregation between CP/CPPS and vasectomy (Figure 3). There were 48 genes with expression $>1.5 \times$ different in CP/CPPS $v s$. controls and a $\mathrm{P}$ value $<0.05$ with greatest differences seen for SLAMF8 (22.3 $\times$ higher, $\mathrm{P}=0.0004)$, LAIR1 (18.8× higher, $\mathrm{P}=0.004)$ and $\mathrm{C} 1 \mathrm{QC}(16.5 \times$ higher, $\mathrm{P}=0.01)$ (Figure 4). Gene set analysis was different for a number of pathways including carbohydrate metabolism (SS 1.8), Neurons and Neurotransmission (SS 1.5), Adaptive Immunity (SS 1.4) and Inflammatory Signaling (SS 1.3). More impressive differences were seen between phenotypes. For Organ Specific (yes $v s$. no) there were 58 genes with significantly different expression led by S100A12 ( $-298 \times$ change, $\mathrm{P}=0.03)$, ILIRN ( $-65.3 \times$ change, $\mathrm{P}=0.008)$ and OSMR $(-49.4 \times, \mathrm{P}=0.004)$. By gene set analysis grouping there were differences in Astrocyte function (SS 1.6), Cytokine signaling (SS 1.5), Innate Immune Response (SS 1.5) and Growth factor signaling (SS 1.4). Neurologic/Systemic had 4 genes with differential expression including S100A12 $(-121 \times$ change, $\mathrm{P}=0.009)$ and STEAP4 $(-26.9 \times$ change, $\mathrm{P}=0.01)$. Gene set analyses did not show distinct groupings.

\section{Discussion}

CP/CPPS is a syndrome characterized by pelvic and genital pain often accompanied by urinary symptoms and sexual dysfunction in the absence of acute or recurrent urinary tract infection (7). No single common etiology appears to explain clinical findings and risk factors (11), with evidence for infection, inflammation, muscle spasm, neuropathic activation and central neurologic changes found depending on the patient population. Lack of uniformity in presentation and response to therapy led to the development of the UPOINT clinical phenotyping system which classifies patients according to 6 clinical domains and guides multimodal therapy based on those findings (10). The domains are clinically diagnosed and have not been externally validated by other biomarkers.

Prior attempts to differentiate CP/CPPS patients from controls by findings or biomarkers have been met with variable success. There is no difference in rates of infection or inflammation (as measured by presence of white cells) in urine or prostatic fluid (12), however there are changes in microbial ecology both in the urine (13) and bowel (14). Several studies have shown differences in specific cytokines between CP/CPPS patients and controls both for expression (3) and genetic polymorphisms (4). Such work has been limited by the number of genes that could be practically analyzed simultaneously. Newer technologies such as NanoString allow the assessment of a large number of genes on relatively small samples. This is especially important since, while a single preselected gene might have a relatively modest increased expression, such modest expression of multiple genes within the same pathway may have a powerful biologic effect. Given the importance of inflammation and neuropathic pain in the proposed etiology of CP/CPPS we chose the premade NanoString neuroinflammation panel for this study.

The first striking finding for gene analysis in our samples was that there were more differences seen between CP/ CPPS patients with different phenotypes than there were between patients and controls. This finding strongly supports the hypothesis that CP/CPPS is not a single disease with a common underlying biology for all or most patients but rather a syndrome with heterogeneous phenotypes that have a different biologic basis (15). The second surprising finding was that the majority of differences in gene expression and biologic pathways was for inflammation 


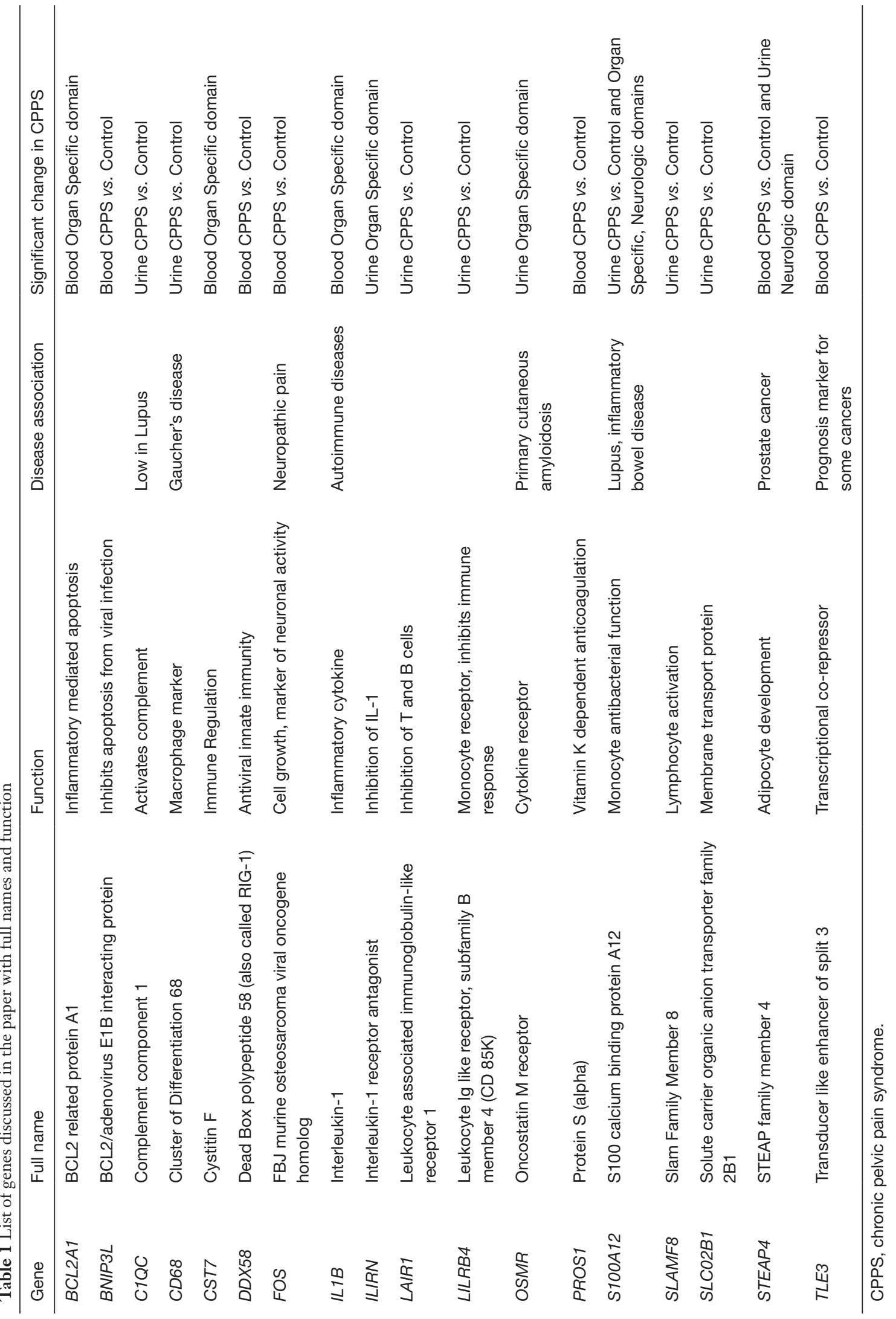


A

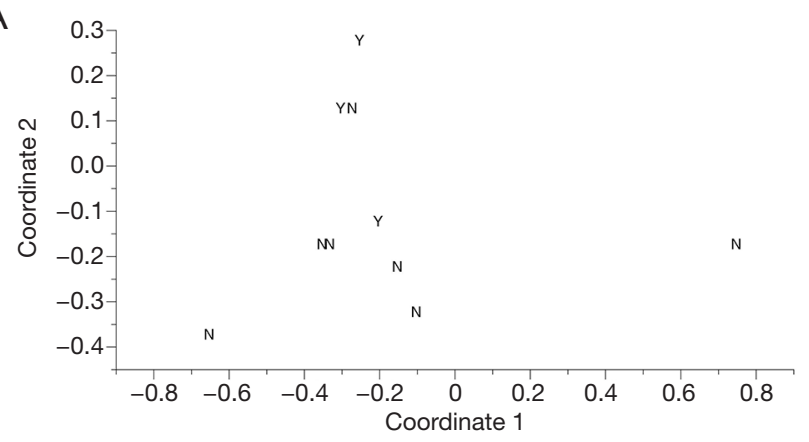

C

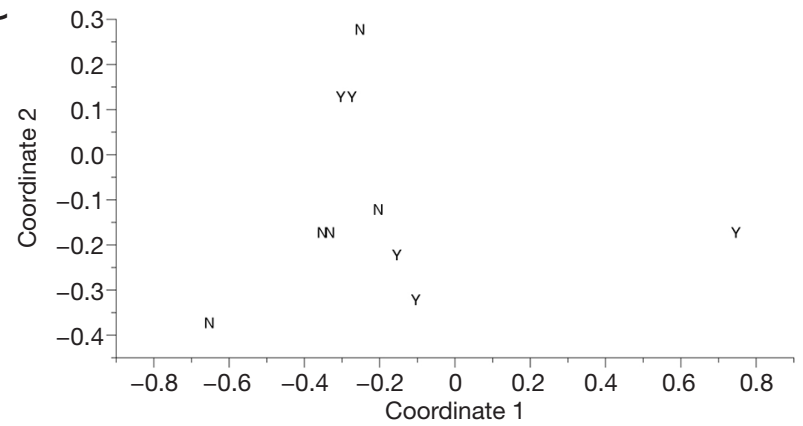

B

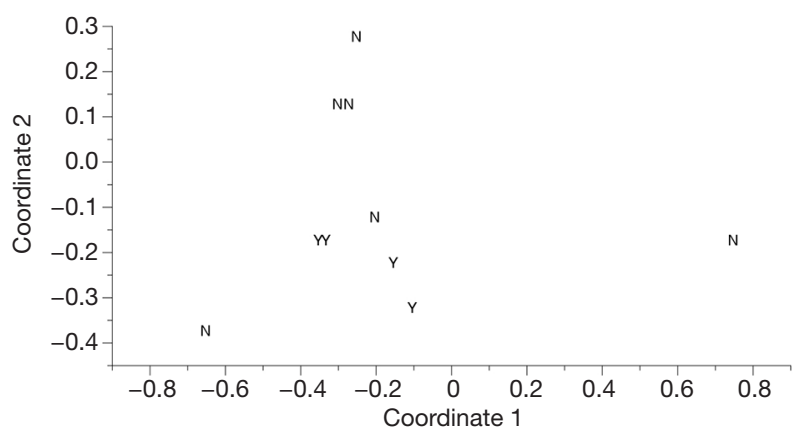

Figure 2 Multidimensional Scaling (MDS) Plots of gene expression for individual chronic prostatitis/chronic pelvic pain syndrome patients in blood by presence of UPOINT domain. Each patient represented by a Y (yes, domain present) or N (no, domain not present). For exactly overlapping data points the 2 symbols were placed side by side. Axis are arbitrary units and overall differences in gene expression are represented by the distance between any two points. (A) Organ Specific Domain. Each patient represented by a Y (yes, Organ specific domain present) or N (no, Organ specific domain not present). (B) Psychosocial Domain Each patient represented by a Y (yes, Psychosocial Domain domain present) or N (no, Psychosocial Domain domain not present). (C) Neurogenic/Systemic Domain Each patient represented by a Y (yes, Neurogenic/Systemic domain present) or N (no, Neurogenic/Systemic domain not present).

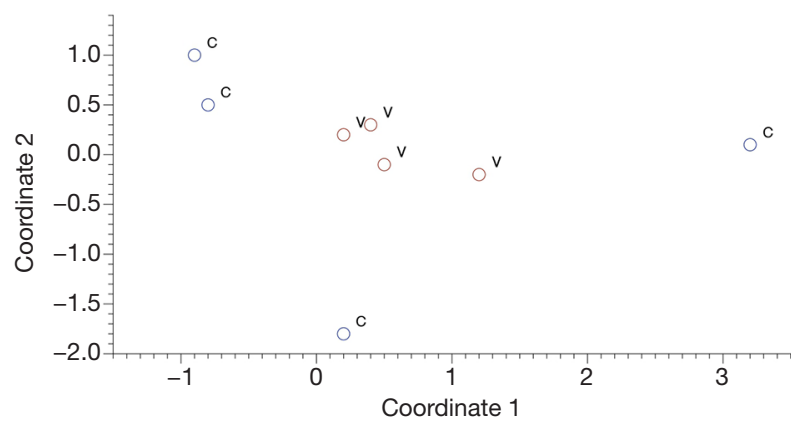

Figure 3 Multidimensional Scaling Plots for chronic prostatitis/ chronic pelvic pain syndrome patients $(\mathrm{C})$ and vasectomy controls $(\mathrm{V})$ in urine. Axis are arbitrary units and overall differences in gene expression are represented by the distance between any two points.

rather than neuropathic pain and none of the neuropathic genes were different for patients with the Neurologic/ Systemic or Psychogenic domains. Of note, there were

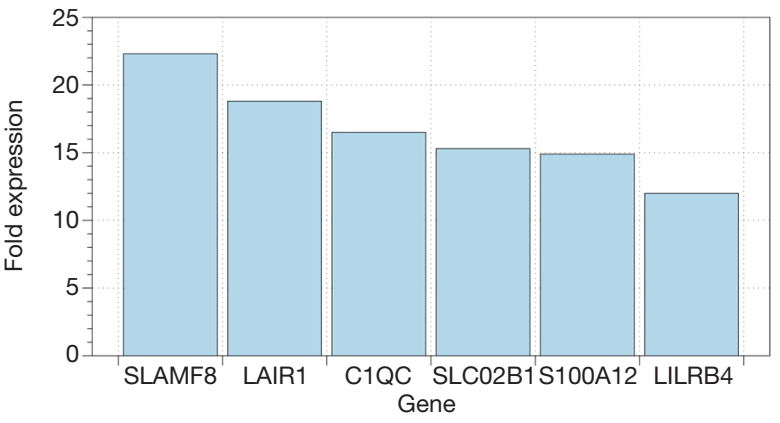

Figure 4 Mean differential gene expression between chronic prostatitis/chronic pelvic pain syndrome patients and controls in urine. Genes listed had at least $10 \times$ differential expression with $\mathrm{P}<0.05$. For full gene name and function please see Table 1 .

patients in the Neurologic/Systemic group with profound systemic symptoms including debilitating fibromyalgia. Even genes strongly associated with neuropathic pain and 
previously found in prostate fluid of CP/CPPS patients such as nerve growth factor (NGF) (5) did not have differential expression in urine or blood. This is counterintuitive to our current thinking of CP/CPPS patients with systemic symptoms having more in common with other centrally mediated neuropathic pain syndromes (16). Indeed, such changes may be present in these patients but not only in organ sites not samples by blood or urine. Furthermore, the presence or transit time of these cells in the blood may make sampling more difficult than cells recovered from the urine.

While the findings in blood did not demonstrate major differences, we did find differential inflammatory and neuropathic gene expression in urine. The greatest changes were seen in genes such as SLAMF8 (17) and LAIR1 (18) which regulate monocytes, T and B cells. Gene set differences included both neurotransmission and inflammation. It has been a central paradox in CP/CPPS that even though "prostatitis" implies inflammation, only a small minority of these patients have inflammation visible on prostate biopsy specimens (19). Nevertheless, inflammatory mediators have been found in the prostate fluid (20) and many patients do respond to anti-inflammatory based therapies (21). Even with a shift in thinking of CP/CPPS as more of a neuromuscular or neuropathic condition, there is persistent evidence of immune cell involvement even if biopsy evidence of inflammation or presence of white cells in expressed prostatic secretions fail to correlate with specific symptoms (22). This can explain in part symptomatic improvement from antibiotics such as quinolones that have immunomodulatory effects (23) and nutraceuticals with antioxidant properties such as quercetin (21) and cernilton (24). Such immunomodulation may be insufficient however without multimodal therapy that addresses both muscular spasm (25) and neuropathic pain (26).

Strengths of this study include the broad range of genes tested, inclusion of a well-matched control group and complete clinical phenotyping of the CP/CPPS patients, a feature lacking in all prior genetic studies. Limitations include the relatively small numbers which did not allow a sufficient heterogeneity in clinical phenotypes to adequately examine the impact of the Urinary symptoms or Tenderness of Muscle domains on genetic expression. We also did not have long term follow up to determine whether specific treatments resulted in success based on the gene expression.

In conclusion, men with CP/CPPS have a diverse set of neuroinflammatory genes with differential expression compared to controls. Furthermore, clinical phenotypes have distinct patterns of gene expression and differences between phenotypes were more pronounced than between patients and controls. Surprisingly, those with systemic symptoms of neuropathic conditions did not show differences in neuropathic genes, at least in the blood. This approach could lead to novel biomarker development and further validates the biologic basic of clinical phenotyping.

\section{Acknowledgments}

This research was funded in part by generous philanthropic support at Cleveland Clinic. Special thanks to Mr. Sam Kellett and Mr. Paul Vitek for their support of our work. Funding: None.

\section{Footnote}

Data Sharing Statement: Available at https://dx.doi. org/10.21037/tau-21-387

Conflicts of Interest: All authors have completed the ICMJE uniform disclosure form (available at https://dx.doi. org/10.21037/tau-21-387). DAS serves as an unpaid editorial board member of Translational Andrology and Urology from Aug 2020 to Jul 2022. DAS consults for Utility Pharmaceuticals and Urogen and has investment interest in Triurol. The other authors have no conflicts of interest to declare.

Ethical Statement: The authors are accountable for all aspects of the work (if applied, including full data access, integrity of the data and the accuracy of the data analysis) in ensuring that questions related to the accuracy or integrity of any part of the work are appropriately investigated and resolved. The study was approved by the Cleveland Clinic IRB (protocol 19-1515) and appropriate written consent was obtained on all subjects. The study conformed to the provisions of the Declaration of Helsinki (as revised in 2013).

Open Access Statement: This is an Open Access article distributed in accordance with the Creative Commons Attribution-NonCommercial-NoDerivs 4.0 International License (CC BY-NC-ND 4.0), which permits the noncommercial replication and distribution of the article with the strict proviso that no changes or edits are made and the original work is properly cited (including links to both the formal publication through the relevant DOI and the license). 
See: https://creativecommons.org/licenses/by-nc-nd/4.0/.

\section{References}

1. DeWitt-Foy ME, Nickel JC, Shoskes DA. Management of Chronic Prostatitis/Chronic Pelvic Pain Syndrome. Eur Urol Focus 2019;5:2-4.

2. Murphy SF, Schaeffer AJ, Done JD, et al. Commensal bacterial modulation of the host immune response to ameliorate pain in a murine model of chronic prostatitis. Pain 2017;158:1517-27.

3. Chen L, Bian Z, Chen J, et al. Immunological alterations in patients with chronic prostatitis/chronic pelvic pain syndrome and experimental autoimmune prostatitis model: A systematic review and meta-analysis. Cytokine 2021;141:155440.

4. Shoskes DA, Albakri Q, Thomas K, et al. Cytokine polymorphisms in men with chronic prostatitis/chronic pelvic pain syndrome: association with diagnosis and treatment response. J Urol 2002;168:331-5.

5. Watanabe T, Inoue $M$, Sasaki $K$, et al. Nerve growth factor level in the prostatic fluid of patients with chronic prostatitis/chronic pelvic pain syndrome is correlated with symptom severity and response to treatment. BJU Int 2011;108:248-51.

6. Geiss GK, Bumgarner RE, Birditt B, et al. Direct multiplexed measurement of gene expression with colorcoded probe pairs. Nat Biotechnol 2008;26:317-25.

7. Krieger JN, Nyberg L Jr, Nickel JC. NIH consensus definition and classification of prostatitis. JAMA 1999;282:236-7.

8. Litwin MS, McNaughton-Collins M, Fowler FJ Jr, et al. The National Institutes of Health chronic prostatitis symptom index: development and validation of a new outcome measure. Chronic Prostatitis Collaborative Research Network. J Urol 1999;162:369-75.

9. Shoskes DA, Nickel JC, Dolinga R, et al. Clinical phenotyping of patients with chronic prostatitis/chronic pelvic pain syndrome and correlation with symptom severity. Urology 2009;73:538-42; discussion 542-3.

10. Shoskes DA, Nickel JC, Kattan MW. Phenotypically directed multimodal therapy for chronic prostatitis/ chronic pelvic pain syndrome: a prospective study using UPOINT. Urology 2010;75:1249-53.

11. Pontari MA. Etiology of chronic prostatitis/chronic pelvic pain syndrome: psychoimmunoneurendocrine dysfunction (PINE syndrome) or just a really bad infection? World J Urol 2013;31:725-32.
12. Nickel JC, Alexander RB, Schaeffer AJ, et al. Leukocytes and bacteria in men with chronic prostatitis/chronic pelvic pain syndrome compared to asymptomatic controls. J Urol 2003;170:818-22.

13. Shoskes DA, Altemus J, Polackwich AS, et al. The Urinary Microbiome Differs Significantly Between Patients With Chronic Prostatitis/Chronic Pelvic Pain Syndrome and Controls as Well as Between Patients With Different Clinical Phenotypes. Urology 2016;92:26-32.

14. Shoskes DA, Wang H, Polackwich AS, et al. Analysis of Gut Microbiome Reveals Significant Differences between Men with Chronic Prostatitis/Chronic Pelvic Pain Syndrome and Controls. J Urol 2016;196:435-41.

15. Samplaski MK, Li J, Shoskes DA. Clustering of UPOINT domains and subdomains in men with chronic prostatitis/ chronic pelvic pain syndrome and contribution to symptom severity. J Urol 2012;188:1788-93.

16. Woodworth D, Mayer E, Leu K, et al. Unique Microstructural Changes in the Brain Associated with Urological Chronic Pelvic Pain Syndrome (UCPPS) Revealed by Diffusion Tensor MRI, Super-Resolution Track Density Imaging, and Statistical Parameter Mapping: A MAPP Network Neuroimaging Study. PLoS One 2015;10:e0140250.

17. Wang G, van Driel BJ, Liao G, et al. Migration of myeloid cells during inflammation is differentially regulated by the cell surface receptors Slamf1 and Slamf8. PLoS One 2015;10:e0121968.

18. Kumawat K, Geerdink RJ, Hennus MP, et al. LAIR-1 Limits Neutrophilic Airway Inflammation. Front Immunol 2019;10:842.

19. True LD, Berger RE, Rothman I, et al. Prostate histopathology and the chronic prostatitis/chronic pelvic pain syndrome: a prospective biopsy study. J Urol 1999;162:2014-8.

20. Shahed AR, Shoskes DA. Correlation of beta-endorphin and prostaglandin E2 levels in prostatic fluid of patients with chronic prostatitis with diagnosis and treatment response. J Urol 2001;166:1738-41.

21. Shoskes DA, Zeitlin SI, Shahed A, et al. Quercetin in men with category III chronic prostatitis: a preliminary prospective, double-blind, placebo-controlled trial. Urology 1999;54:960-3.

22. Schaeffer AJ, Knauss JS, Landis JR, et al. Leukocyte and bacterial counts do not correlate with severity of symptoms in men with chronic prostatitis: the National Institutes of Health Chronic Prostatitis Cohort Study. J Urol 2002;168:1048-53. 
23. Dalhoff A, Shalit I. Immunomodulatory effects of quinolones. Lancet Infect Dis 2003;3:359-71.

24. Wagenlehner FM, Schneider H, Ludwig M, et al. A pollen extract (Cernilton) in patients with inflammatory chronic prostatitis-chronic pelvic pain syndrome: a multicentre, randomised, prospective, double-blind, placebo-controlled phase 3 study. Eur Urol 2009;56:544-51.

25. Shoskes DA, Berger R, Elmi A, et al. Muscle tenderness

Cite this article as: Shoskes DA, Keslar KS, Gotwald P, Berglund R, Vij S. Neuroinflammatory gene expression in chronic prostatitis/chronic pelvic pain syndrome patients: insights into etiology and phenotype biology. Transl Androl Urol 2021;10(8):3340-3347. doi: 10.21037/tau-21-387 in men with chronic prostatitis/chronic pelvic pain syndrome: the chronic prostatitis cohort study. J Urol 2008;179:556-60.

26. Pontari MA, Krieger JN, Litwin MS, et al. Pregabalin for the treatment of men with chronic prostatitis/chronic pelvic pain syndrome: a randomized controlled trial. Arch Intern Med 2010;170:1586-93. 